\title{
Iterative Learning Control of a Flexible Mechanical System Using Accelerometers
}

\author{
Svante Gunnarsson, Mikael Norrlöf \\ Department of Electrical Engineering \\ Linköping University, S-581 83 Linköping, Sweden \\ www: http://www.control.isy.liu.se \\ email: svante@isy.liu.se, mino@isy.liu.se
}

2000-05-31

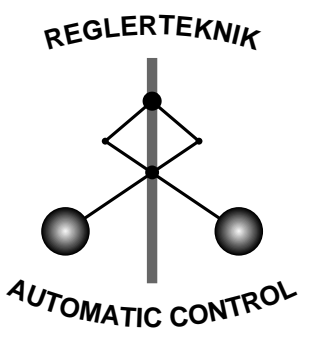

LINKÖPING

To be presented at the 6th IFAC Symposium on Robot Control SYROCO '00, Vienna, Austria, September 2000. Technical reports from the Automatic Control group in Linköping are available by anonymous ftp at the address 130.236.20.24 (ftp.control.isy.liu.se/pub/Reports/). This report is contained in the compressed postscript file 2256.ps.gz. 


\title{
ITERATIVE LEARNING CONTROL OF A FLEXIBLE MECHANICAL SYSTEM USING ACCELEROMETERS $^{1}$
}

\author{
Svante Gunnarsson and Mikael Norrlöf* \\ * Department of Electrical Engineering, Linköping University \\ SE-58183 Linköping, Sweden \\ Email:svante@isy.liu.se,mino@isy.liu.se
}

\begin{abstract}
Control of a flexible mechanical system using Iterative Learning Control (ILC) is studied using a linear two-mass model. The available signals are position of the first mass and acceleration of the second mass. An ILC algorithm using an estimate of the position of the second mass is evaluated in simulations showing promising properties.
\end{abstract}

Keywords: iterative improvement, learning algorithms, flexible arm, robot control, accelerometers

\section{INTRODUCTION}

The aim of this paper is to present some initial experiences from the use of accelerometers in ILC applied to flexible mechanical systems. This will be done by presenting a simulation study where ILC is used to control the motion of the second mass in a system consisting of two masses connected via a spring and a damper. Different learning strategies applied to flexible mechanical systems has been studied previously in e.g. (Panzieri and Ulivi 1995), (Velthuis et al. 1996) and (Lange and Hirzinger 1999). In these papers it is assumed that the position (angle) of the mass to be controlled can be measured. Here instead it is assumed that only the acceleration of the mass to be controlled can be measured. In (Miyazaki et al. 1986), which also deals with a flexible system, an acceleration signal is used in an ILC algorithm. The way it is used and the ILC algorithm is designed differ however completely from the approach presented here.

\footnotetext{
1 This work was supported by CENIIT at Linköping University and by ABB Robotics within ISIS at Linköping University.
}

The presentation will be restricted to discretetime, linear SISO systems. The discrete-time approach is logical since the ILC algorithm operates using sampled data. The restriction to linear systems may seem restrictive when industrial robots are the main application area of ILC. In the approach taken here it is however assumed that ILC is used as a complement to an existing robot control system that reduces the nonlinear effects.

\section{THE ILC ALGORITHM}

In this paper an ILC algorithm based on optimization will be used. This approach has previously been considered in e.g (Gorinevsky et al. 1995), (Lee and Lee 1998) and (Frueh and Phan 1998). This algorithm can however be interpreted as a more conventional filter based algorithm, as described in (Gunnarsson and Norrlöf 1999) and (Gunnarsson and Norrlöf 2000). 
Introduce the vectors

$$
\begin{aligned}
\mathbf{Y}_{k} & =\left(y_{k}(0), \ldots, y_{k}(N)\right)^{T} \\
\mathbf{U}_{k} & =\left(u_{k}(0), \ldots, u_{k}(N)\right)^{T} \\
\mathbf{V}_{k} & =\left(v_{k}(0), \ldots, v_{k}(N)\right)^{T} \\
\mathbf{R} & =(r(0), \ldots, r(N))^{T} \\
\mathbf{E}_{k} & =\mathbf{R}-\mathbf{Y}_{k}
\end{aligned}
$$

where $u_{k}(t)$ and $y_{k}(t)$ denote the ILC input signal and the output signal respectively. Furthermore $r(t)$ and $v_{k}(t)$ denote the desired output (reference) signal and a vector of disturbance signals respectively. The subscript $k$ denotes iteration number. Using these notations the system can be described by the equation

$$
\mathbf{Y}_{k}=\mathbf{T}_{r} \mathbf{R}+\mathbf{T}_{u} \mathbf{U}_{k}+\mathbf{T}_{v} \mathbf{V}_{k}
$$

where $\mathbf{T}_{u}$ is the lower triangular matrix formed by the impulse response coefficients of the transfer operator $T_{u}(q)$ from ILC input signal $u_{k}(t)$ to output signal $y_{k}(t)$ and $\mathbf{T}_{r}$ and $\mathbf{T}_{v}$ are defined analogously. Equation (6) is a general formulation that covers systems working in open loop, where $u_{k}(t)$ represents the actual system input, as well as systems working in closed loop, where the signal $u_{k}(t)$ is added to the control signal generated by the conventional control system. The difference in conditions is reflected in different definitions of the matrices in equation (6). Consider now the criterion

$$
\mathbf{J}=\mathbf{E}_{k+1}^{T} \mathbf{W}_{e} \mathbf{E}_{k+1}+\mathbf{U}_{k+1}^{T} \mathbf{W}_{u} \mathbf{U}_{k+1}
$$

where $\mathbf{W}_{e}$ and $\mathbf{W}_{u}$ are weight matrices determining the trade off between performance and input energy. The criterion is minimized subject to the constraint

$$
\left(\mathbf{U}_{k+1}-\mathbf{U}_{k}\right)^{T}\left(\mathbf{U}_{k+1}-\mathbf{U}_{k}\right) \leq \delta
$$

The optimal input vector is updated according to

$$
\mathbf{U}_{k+1}=\mathbf{Q}\left(\mathbf{U}_{k}+\mathbf{L} \mathbf{E}_{k}\right)
$$

where

$$
\begin{aligned}
\mathbf{Q} & =\left(\mathbf{W}_{u}+\lambda \cdot \mathbf{I}+\mathbf{T}_{u}^{T} \mathbf{W}_{e} \mathbf{T}_{u}\right)^{-1} \\
& \times\left(\lambda \cdot \mathbf{I}+\mathbf{T}_{u}^{T} \mathbf{W}_{e} \mathbf{T}_{u}\right)
\end{aligned}
$$

and

$$
\mathbf{L}=\left(\lambda \cdot \mathbf{I}+\mathbf{T}_{u}^{T} \mathbf{W}_{e} \mathbf{T}_{u}\right)^{-1} \mathbf{T}_{u}^{T} \mathbf{W}_{e}
$$

The updating matrices $\mathbf{Q}$ and $\mathbf{L}$ hence depend on the nominal model $\mathbf{T}_{u}$ which is considered to be given and the weight matrices $\mathbf{W}_{u}$ and $\mathbf{W}_{e}$. The Lagrange multiplier $\lambda$ is not computed explicitly but instead used as a design variable. As illustrated in (Gunnarsson and Norrlöf 1999) a nominal model of the closed loop system, represented by $\mathbf{T}_{u}$ can be obtained using system identification.

\section{A FLEXIBLE SYSTEM}

The main interest in this paper is the application of ILC to systems containing flexibilities. As an example of this situation a linear model of a flexible servo will be studied, as shown in Figure 1. Here $\theta_{m}(t)$ and $\theta_{a}(t)$ denote motor angle and load angle respectively and $u$ is the applied torque, which is the input signal. This can be viewed as a simplified description of a one link robot arm with the flexibility concentrated to the joint. It is assumed that the system is working in a position where the gravitational effects can be neglected.

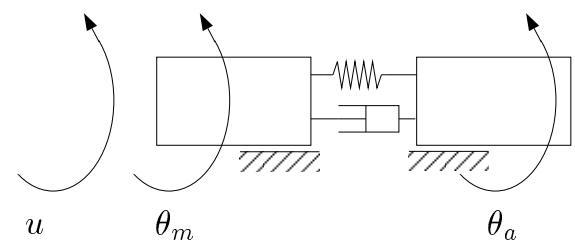

Fig. 1. Two-mass model.

Torque balances yield the following equations

$$
J_{m} \ddot{\theta}_{m}+f_{m} \dot{\theta}_{m}+k\left(\theta_{m}-\theta_{a}\right)+d\left(\dot{\theta}_{m}-\dot{\theta}_{a}\right)=u
$$

and

$$
J_{a} \ddot{\theta}_{a}+f_{a} \dot{\theta}_{a}-k\left(\theta_{m}-\theta_{a}\right)-d\left(\dot{\theta}_{m}-\dot{\theta}_{a}\right)=0
$$

where $J_{m}$ and $J_{a}$ denote the moment of inertia of each mass while $f_{m}$ and $f_{a}$ denote the viscous friction coefficient of each mass. Finally $k$ and $d$ denote the stiffness and damping respectively of the flexibility between the two masses. Using the state variables

$$
x_{1}=\theta_{m} \quad x_{2}=\dot{\theta}_{m} \quad x_{3}=\theta_{a} \quad x_{4}=\dot{\theta}_{a}
$$

the system can be described on state space form as

$$
\dot{x}=A x+B u \quad y=C x=\left(\begin{array}{c}
\theta_{m} \\
\ddot{\theta}_{a}
\end{array}\right)
$$

since the available measurements are position of the first mass and acceleration of the second mass.

It is important to note that the main control goal is that the load angle $\theta_{a}$ shall follow a desired trajectory given by Figure 2. The normal case in many robot systems is that only the motor angle $\theta_{m}$ can be measured. Measurements of the load angle (position) are normally not available. The aim here is to investigate how the addition of an accelerometer measuring the load acceleration can be used in an ILC algorithm for improving the servo performance. The system is controlled using a conventional two-degrees of freedom controller structure giving the servo performance shown in Figure 2. The aim in the remaining part of this paper is to investigate how ILC can be used to 
improve the servo properties both with respect to speed and damping. In the simulations presented below the ILC signal is added to the original reference signal, which means that the ILC iterations generate a stepwise reshaping of the reference signal. The idea of reshaping the reference signal can also be found in (Lee et al. 1997). It is also possible to add the ILC input to the control signal generated by the existing control system, which means that the ILC input will act as a torque feed-forward signal.

\section{ESTIMATING THE LOAD ANGLE}

The main idea in the paper is to use an estimate of the load angle in the ILC algorithm. The estimate is formed as a combination of load acceleration and motor angle by using a a state estimator based on the nominal relationship between the motor and load angles. Recall equation (13)

$$
J_{a} \ddot{\theta}_{a}+f_{a} \dot{\theta}_{a}-k\left(\theta_{m}-\theta_{a}\right)=0
$$

where for simplicity the damping coefficient $d$ is put to zero. Introducing the state variables $\bar{x}_{1}=\theta_{a}$ and $\bar{x}_{2}=\dot{\theta}_{a}$ and the measured signal $\bar{y}(t)=\ddot{\theta}_{a}$ equation (16) can be expressed in state space form as

$$
\dot{\bar{x}}=\bar{A} \bar{x}+\bar{B} \theta_{m} \quad \bar{y}=\bar{C} \bar{x}+\bar{D} \theta_{m}
$$

A Kalman filter for this system is then given by

$$
\dot{\bar{x}}=\bar{A} \hat{\bar{x}}+\bar{B} \theta_{m}+K\left(\bar{y}-C \hat{\bar{x}}-\bar{D} \theta_{m}\right)
$$

The estimated load angle can then be expressed using transfer functions as

$$
\hat{\Theta}_{a}(s)=\hat{\bar{X}}_{1}(s)=F_{\bar{y}}(s) \bar{Y}(s)+F_{\theta_{m}}(s) \Theta_{m}(s)
$$

where

$$
F_{\bar{y}}(s)=\left(\begin{array}{ll}
1 & 0
\end{array}\right)(s I-\bar{A}+K \bar{C})^{-1} K
$$

and

$$
F_{\theta_{m}}(s)=\left(\begin{array}{ll}
1 & 0
\end{array}\right)(s I-\bar{A}+K \bar{C})^{-1}(\bar{B}-K \bar{D})
$$

When designing the Kalman filter the variance $R_{2}$ of the load acceleration signal can be used as a design variable that affects the balance between the measured load acceleration and the measured motor angle. Choosing a high value of $R_{2}$ implies that the load acceleration is almost neglected. The resulting filter from $\theta_{m}$ to $\hat{\theta}_{a}$ is then almost exactly the transfer function obtained from equation (16). The load angle estimate is then obtained by feeding the motor angle through the nominal transfer function from motor angle to load angle. In the nominal case this of course gives a good estimate of the load angle but the estimate is very sensitive to model errors. For example, a $20 \%$ error in $J_{a}$ gives approximately $10 \%$ change in $\omega_{a}=\sqrt{k / J_{a}}$, determining the location of the peak in the transfer function. On the other hand, choosing a low value of $R_{2}$ means that the load acceleration plays a large role in the estimation of load angle. The filters $F_{\bar{y}}$ and $F_{\theta_{m}}$ for this case are shown in Figure 3. In the considered frequency range the gain of the filters is almost constant. Considering equation (16) this behavior is logical since a slight reformulation of equation (16) gives

$$
\theta_{a}=\theta_{m}-\frac{J_{a}}{k} \ddot{\theta}_{a}-\frac{f_{a}}{k} \dot{\theta}_{a}
$$

With good measurements of $\ddot{\theta}_{a}$ available an approximation of the load angle is given by

$$
\hat{\theta}_{a}=\theta_{m}-\frac{\hat{J}_{a}}{\hat{k}} \ddot{\theta}_{a}
$$

where the effect of the viscous friction has been neglected. Furthermore $\hat{k}$ and $\hat{J}_{a}$ denote estimates of the spring constant and moment of inertia. The left part of Figure 3 hence correspond to the constant $-\frac{\hat{J}_{a}}{\hat{k}}$ while the right part correspond to the unit factor in front of $\theta_{m}$. In order to analyze how sensitive the estimation procedure is the following calculations can be made. The relationship between the motor angle and the load angle is, using (13), given by

$$
\Theta_{a}(s)=G_{a}(s) \Theta_{m}(s)
$$

The measured load acceleration can therefore be expressed

$$
\bar{Y}(s)=s^{2} G_{a}(s) \Theta_{m}(s)
$$

Using equation (23) this gives

$$
\hat{\Theta}_{a}(s)=\left(1-\frac{\hat{J}_{a}}{\hat{k}} s^{2} G_{a}(s)\right) \Theta_{m}(s)
$$

The transfer function

$$
\hat{G}_{a}(s)=\left(1-\frac{\hat{J}_{a}}{\hat{k}} s^{2} G_{a}(s)\right)
$$

giving the estimated load angle can now be compared with the transfer function $G_{a}(s)$ giving the true load angle. Figure 4 shows the Bode diagram of $G_{a}(s)$ and $\hat{G}_{a}(s)$ for some different values of $\hat{J}_{a}$. Around the resonance frequency the true $G_{a}(s)$ and the estimate $\hat{G}_{a}(s)$ are very close while they differ more for higher frequencies. It can also be seen that the gain of $\hat{G}_{a}(s)$ is systematically higher than for $G_{a}(s)$. It therfore seems reasonable to low-pass filter the load angle estimate before using it in the ILC algorithm.

\section{ILC USING ESTIMATED LOAD ANGLE ERROR}

The idea presented above will now be evaluated in some simulations. The ILC input is computed as

$$
\mathbf{U}_{k+1}=\mathbf{Q}\left(\mathbf{U}_{k}+\mathbf{L} \hat{\mathbf{E}}_{k}\right)
$$


where $\hat{\mathbf{E}}_{k}$ is the error between load angle reference and estimated load angle.

In the first simulation example (the nominal case) it is assumed that the correct value of the ratio $J_{a} / k$ is available and can be used in equation (23). The design variables are chosen as $\mathbf{W}_{e}=\mathbf{I}, \mathbf{W}_{u}=10^{-2} \cdot \mathbf{I}$ and $\lambda=10^{-2}$. Figure 5 shows the maximum absolute value of the error during 20 iterations. Figure 6 shows the reference load angle and achieved load angle after the last iteration.

In the next simulations the value of $J_{a}$ differs from the nominal value used when designing the ILC algorithm. In the simulations the value of $\hat{J}_{a}$ is $20 \%$ larger/smaller than the true one. Figure 7 shows the evolution of the max error during the iterations in these two cases. The convergence is somewhat affected and the error settles at a slightly higher value.

Finally, the robustness against unmodeled dynamics is evaluated by applying the ILC algorithm to a system of higher order. The higher order system is obtained by splitting the second mass into two parts, each having moment of inertia $J_{a} / 2$. The two masses are connected via a spring with spring constant $4.5 \cdot k$. Both masses are affected by viscous friction with friction constant $f_{a}$. It is assumed that the angle of interest is the angle of the third mass and that the corresponding acceleration is measured. Straightforward application of the ILC algorithm used above immediately leads to the well known behavior of ILC algorithms that the size of the error initially decreases when the low frequency components of the error dominate, while it after some iterations starts to grow when the high frequency components begin dominating. Since the performance requirements in the nominal case were high the instability was not so surprising. In order to improve the robustness of the ILC algorithm a zero-phase low pass filter is applied to the estimated load error. A low pass filtering can also be obtained by increasing $\lambda$ but the cut-off frequency and roll-off of the low pass filtering depend on the nominal model and can hence not be chosen freely. Instead a second order low pass-filter with cut-off frequency $6.25 \mathrm{~Hz}$ ( $0.05 \%$ of the Nyquist frequency) is applied to the estimated load angle error. Since the filtering is carried out off-line it can be done as a zero-phase filtering. The evolution of the error is shown in Figure 8.

\section{ROBUSTNESS CONSIDERATIONS}

The ILC algorithm using estimated load position has so far only been evaluated in one particular example, and it is therefore difficult to draw any general conclusions about the properties of the method. It can however still be of interest to point out some of the robustness aspects.

A standard frequency domain sufficient convergence condition in ILC is

$$
\left|Q\left(e^{i \omega T}\right)\right|\left|1-L\left(e^{i \omega T}\right) T_{u}\left(e^{i \omega T}\right)\right|<1
$$

where $Q(q)$ and $L(q)$ are the filters in the ILC update equation

$$
u_{k+1}(t)=Q(q)\left(u_{k}(t)+L(q) e_{k}(t)\right)
$$

The matrices $\mathbf{Q}$ and $\mathbf{L}$ resulting from the optimization approach can be given a frequency domain interpretation as shown in (Gunnarsson and Norrlöf 1999) and (Gunnarsson and Norrlöf 2000).

Equation (29) is obtained by considering the homogeneous part of a difference equation, in iteration index $k$, for the error $e_{k}(t)$. In the algorithm studied in this paper there are two candidates for such an analysis. The most logical alternative would be to study $e_{k}(t)=r(t)-\theta_{a, k}(t)$, but it turns out to be simpler to instead study $\hat{e}_{k}(t)=r(t)-\hat{\theta}_{a, k}(t)$. If the load angle can be measured the transfer function $T_{u}(q)$ represents the true transfer function between ILC input and and the load angle. This transfer function can then be written

$$
T_{u}(q)=G_{c m}(q) G_{a}(q)
$$

where $G_{c m}(q)$ is the transfer operator from ILC input to motor angle $\theta_{m}(t)$, and as before $G_{a}(q)$ is the transfer operator from motor angle to load angle. Correspondingly the transfer function from ILC input to estimated load angle can be written

$$
\hat{T}_{u}(q)=G_{c m}(q) \hat{G}_{a}(q)
$$

The estimated load angel can hence be written

$$
\hat{\theta}_{a, k}(t)=G_{c m}(q) \hat{G}_{a}(q) u_{k}(t)
$$

Then

$$
\begin{aligned}
& \hat{e}_{k+1}(t)=r(t)-\hat{T}_{u}(q) u_{k+1}(t) \\
= & r(t)-Q(q) \hat{T}_{u}(q) u_{k}(t)-Q(q) L(q) \hat{T}_{u}(q) \hat{e}_{k}(t) \\
= & (1-Q(q)) r(t)+Q(q)\left(1-L(q) \hat{T}_{u}(q)\right) \hat{e}_{k}(t)
\end{aligned}
$$

Hence the corresponding convergence criterion is

$$
\left|Q\left(e^{i \omega T}\right)\right|\left|1-L\left(e^{i \omega T}\right) \hat{T}_{u}\left(e^{i \omega T}\right)\right|<1
$$

To illustrate this condition Figure 9 shows the left hand side of equation (29) and the left hand side of equation (35) using the nominal value $\hat{J}_{a}=J_{a}$ together with the corresponding curves for the cases $\hat{J}_{a}=1.2 \cdot J_{a}$ and $\hat{J}_{a}=0.8 \cdot J_{a}$. Further evaluation of the criterion predicts that $\hat{J}_{a}$ has to be chosen in the interval $0.75 \cdot J_{a}<\hat{J}_{a}<1.4 \cdot J_{a}$ in order to satisfy equation (35). This prediction agrees very well with simulations. By reducing the performance requirements the stable interval for $\hat{J}_{a}$ is increased. 


\section{CONCLUSIONS AND COMMENTS}

ILC has been applied to a flexible mechanical system. An ILC algorithm that uses estimated load angle has shown promising properties in simulations. The load angle is estimated using a Kalman filter where the load acceleration is regarded as system output and motor angle is regarded as system input.

The results in the paper are based on an idealized description of the accelerometer and further work is needed in order to evaluate the sensitivity against measurement and load disturbances. Since the considered model is very simple it is of interest to investigate if the approach can be used on systems with higher order dynamics.

\section{REFERENCES}

Frueh, J.A. and M. Q. Phan (1998). "Linear Quadratic Optimal Learning Control (LQL)". In: Proc. of the 37th IEEE Conference on Decision and Control. Tampa, Florida. pp. 678-683.

Gorinevsky, D.M., D. Torfs and A.A. Goldenberg (1995). "Learning approximation of feedforward dependence on the task parameters: Experiments in direct-drive manipulator tracking". In: Proc. ACC 1995. Seattle, Washington. pp. 883-887.

Gunnarsson, S. and M. Norrlöf (2000). "On the Design of ILC Algorihtms Using Optimization". Technical report. LiTH-ISY-R2209, Department of Electrical Engineering, Linköping University. Linköping, Sweden.

Gunnarsson, S. and M. Norrlöf (1999). "Some Aspects of an Optimization Approach to Iterative Learning Control". In: Proc of the 38th IEEE Conference on Decision and Control. Phoenix, Arizona.

Lange, F. and G. Hirzinger (1999). "Learning Accurate Path Control of Industrial Robots with Joint Elasticity". In: Proc. IEEE Conference on Robotics and Automation. Detroit, MI. pp. 2084-2089.

Lee, J.H., B.H. Lee, S.M. Lee and C.Y. Chung (1997). "Preshaped Trajectory Command for Fast Repetative PTP Motion of PD-Controlled Flexible Joint Manipulators". In: IEEE/RSJ/GI Conference on Intelligent Robots and Systems. Grenoble, France. pp. $1546-1552$.

Lee, K.S. and J.H. Lee (1998). Design of Quadratic Criterion-Based Iterative Learning Control. In Iterative Learning Control: Analysis, Design, Integration and Applications. Z. Bien and J.X. Xu., eds.. Kluwer Academic Publishers.
Miyazaki, F., S. Kawamura, M. Matsumori and S. Arimoto (1986). "Learning Control Scheme for a Class of Robots with Elasticity". In: Proc. of the 25th IEEE Conference on Decision and Control. Athens, Greece. pp. 74-79.

Panzieri, S. and G. Ulivi (1995). "Disturbance rejection of Iterative Learning Control Applied to Trajectory for a Flexible Manipulator". In: Proc. ECC 1995. Rome, Italy. pp. 2374-2379. Velthuis, W.J.R., T.J.A. de Vries and J. van Amerongen (1996). "Learning feed-forward control of a flexible beam". In: Proc. 1996 IEEE Symposium on Intelligent Control. Dearborn, MI. pp. 103-108.

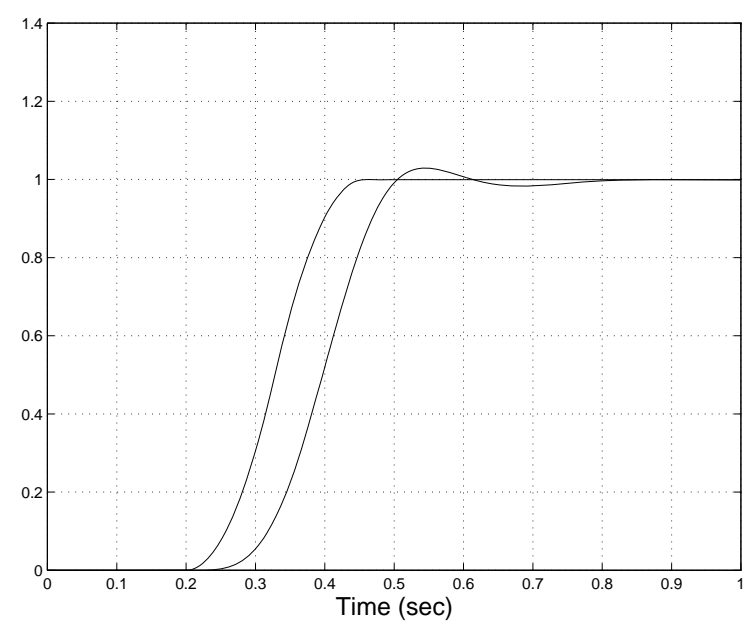

Fig. 2. Load angle reference and load angle without ILC.

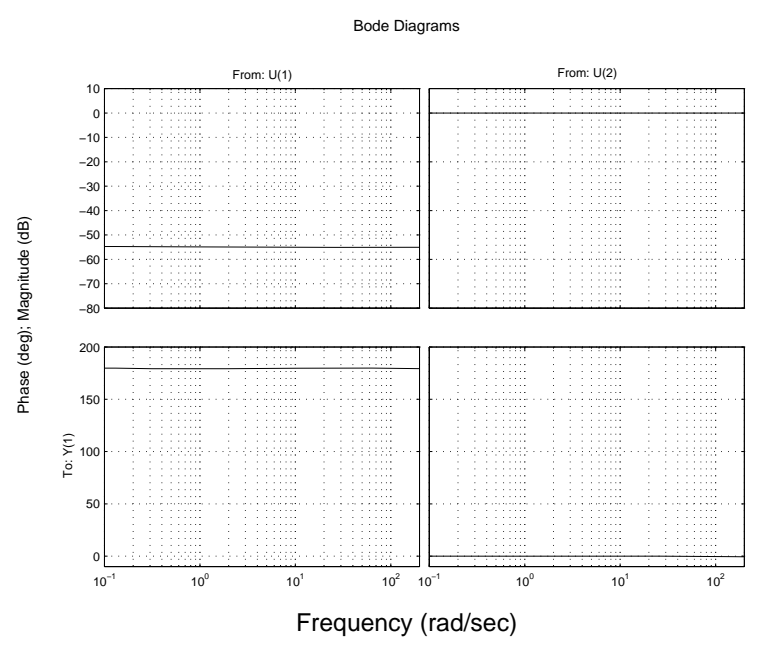

Fig. 3. Estimator filter for low values of $R_{2}$. Left column: $\left|F_{\bar{y}}(i \omega)\right|$ and $\arg F_{\bar{y}}(i \omega)$. Right column: $\left|F_{\theta_{m}}(i \omega)\right|$ and $\arg F_{\theta_{m}}(i \omega)$. 


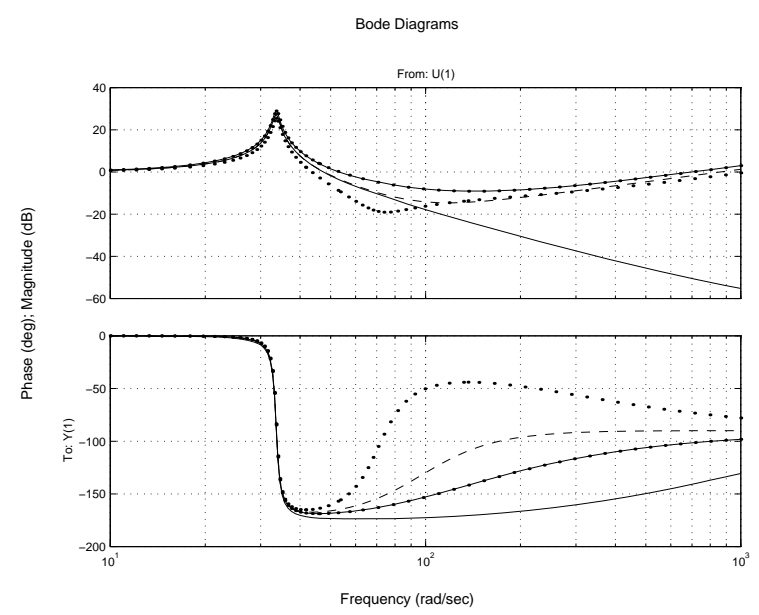

Fig. 4. $G_{a}(s)$ (solid) and $\hat{G}_{a}(s)$ (dashed $\hat{J}_{a}=J_{a}$, dotted $\hat{J}_{a}=1.2 J_{a}$ and dash-dotted $\hat{J}_{a}=$ $0.8 J_{a}$.)

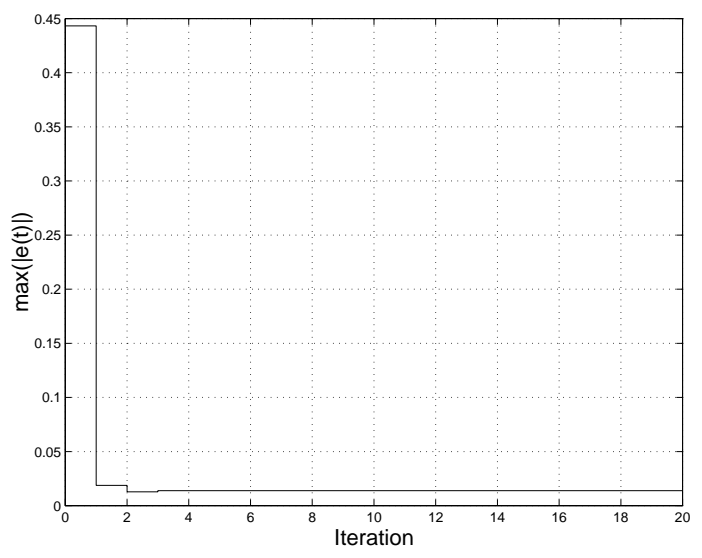

Fig. 5. Max error vs. iteration. $\hat{J}_{a}=J_{a}$.

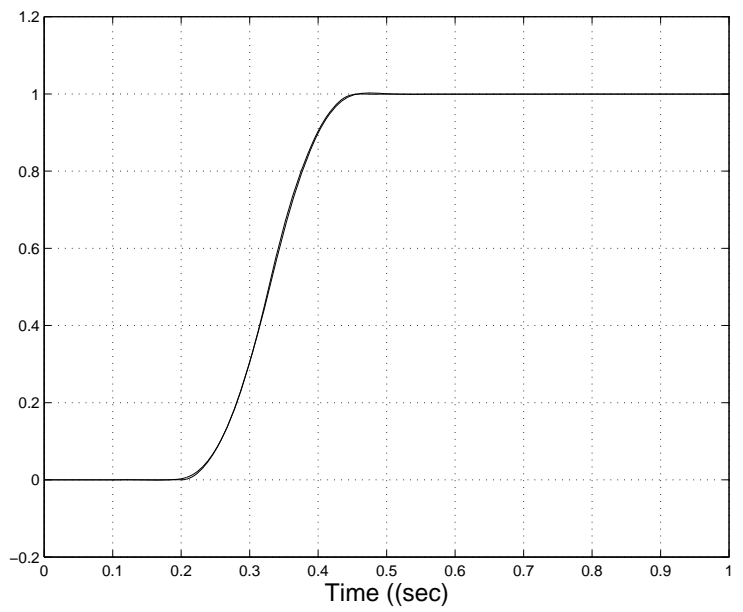

Fig. 6. Load reference angle and load angle after 20 iterations.

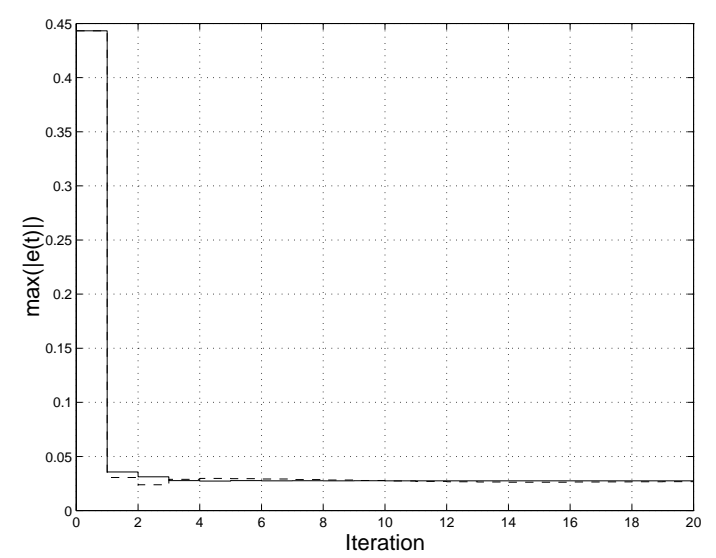

Fig. 7. Max error vs. iteration. Solid $\hat{J}_{a}=1.2 J_{a}$. Dashed $\hat{J}_{a}=0.8 J_{a}$.

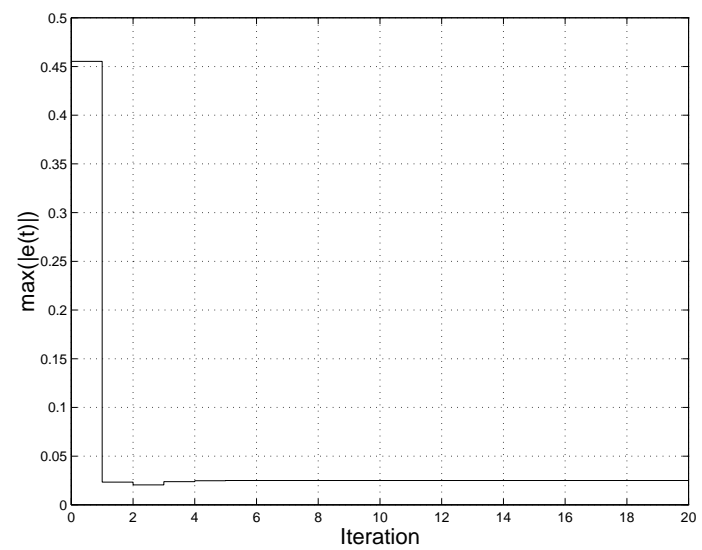

Fig. 8. Max error vs. iteration. Sixth order system.

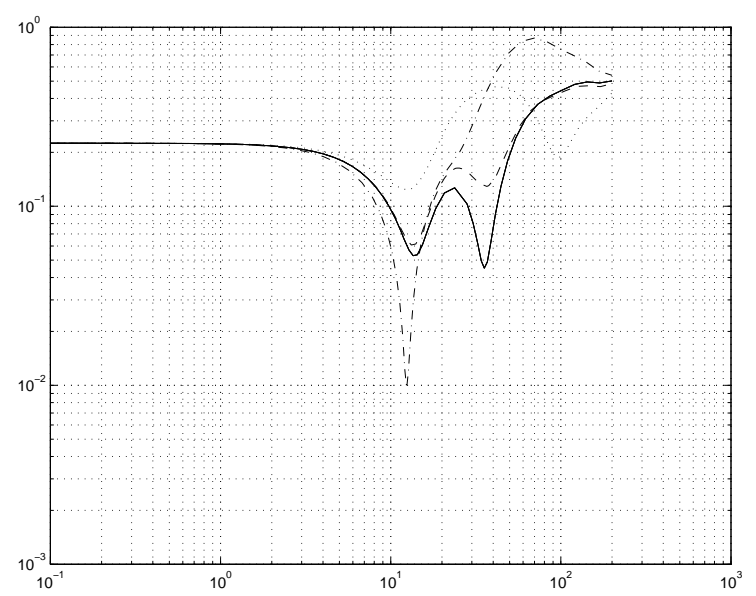

Fig. 9. Solid: Left hand side of equation (29). Left hand side of (35) with $\hat{J}_{a}=J_{a}$ (dashed), $\hat{J}_{a}=0.8 J_{a}$ (dash-dotted) and $\hat{J}_{a}=1.2 J_{a}$ (dotted). 